\title{
Application of Positive Psychology in College Physical Teaching
}

\author{
Wenwen Wang
}

\author{
Jingzhou Institute of Technology, Jingzhou Hubei 434200, China
}

\begin{abstract}
Keywords: College physical education, Positive psychology, Teaching research.
\end{abstract}
\begin{abstract}
Physical education is as an interdisciplinary activity integrating theory and practice, physical and psychological training. It is the foundation for students to engage in other interdisciplinary activities, but also a platform for fostering modern sports talents. However, college education at this stage does not attach enough importance to sports subject, and has not formed a strong research system from physical construction, teaching to the evaluation. Faced with sports course, students are likely to be tired due to laziness, or being afraid of difficulties, and then give up sports activities. Positive psychology is the new idea in physical teaching recently, with a more open and appreciative eyes to see human potential motivation and ability. Based on college physical education, this paper discusses the value of positive psychology and strategies, to provide a reference for the development of college physical education.
\end{abstract}

\section{Introduction}

With the increasingly growing trend of social development and competition, people's psychological pressure is gradually rising. For students at this stage, they need to withstand heavy academic pressure of college life, but also highly focus on intense concentration for getting jobs. However, most college students do not have completely mature mind, relatively lacking the ability to deal with problems, and control emotions, etc. If without timely guidance and intervention, the dual pressures can easily cause students' psychological sub-health state, and even lead to serious accidents dangerous to society, such as suicide and homicide, which will have serious constraints and obstacles for the students' future self-development and embodiment of their own social values ${ }^{[1-3]}$. Positive psychology as an important concept in psychology teaching has a high degree of fitting with college physical education. Based on the author's own experience in teaching, this paper discusses positive psychology and college physical education, and hopes that the majority of teachers and scholars to share and discuss.

\section{Positive Psychology and Its Role in College Physical Education}

Positive psychology, namely a new school of psychology using existing experimental methods and measurement instruments of psychology, to study human strength, virtues and other positive aspects. For the study subject, positive psychology studies average-level persons, and it requires psychologists to use a more open and appreciative look on human potential motivation and ability. Positive psychology advocates psychology should make much research on how ordinary people better develop and live under good conditions, and how people with gift put their potential into full play and other aspects ${ }^{[4]}$. In general, positive psychology is aimed to help people treat mental illness, make people's lives more enriched in a good state of mind, and discover and cultivate talented persons. Study of positive psychology is divided into three levels: first, at the subjective level is the study of positive subjective experience, including happiness and satisfaction, hope and optimism. Secondly, at the personal level is the study of positive personal qualities, including ability to love, ability to work, courage, interpersonal skills, beauty sensibility, perseverance, tolerance, creativity, focus on the future, spirituality, talent and wisdom, etc. In addition, at the level of group, it studies civic virtue and social organizations making individuals become citizens with self-responsibility, altruism, courtesy, tolerance, and professional ethics, including healthy family, good community, efficient schools, and socially responsible media. College physical education, relating to physical and mental aspect, should 
make full use of positive psychology purpose, content and strategies for teaching design. Under good psychological guidance and expectation, students can not only more actively participate in sports activities, and will experience, comprehend and develop in practice.

\section{Status Analysis of College Physical Education}

Since the examination-oriented education ideology is deeply rooted in the field of education, college physical education is facing an unprecedented recession. First, teachers' enthusiasm for teaching is at a low level, and physical education has not been properly developed; in addition, in order to obtain the necessary scores, students are forced to participate in physical examination, resulting in emotional burnout after a long term, which is not conducive to students' physical and mental health ${ }^{[5-8]}$. To further understand the current situation of physical education in the college, based on teaching practice, the author makes the following summary after review of related literature.

Physical education teachers lack sufficient teaching quality ability and research spirit. First, college physical education teachers lack the necessary sense of responsibility in teaching. Responsibility belongs to the category of professional ethics, and it is the necessary psychological basis for active teaching, also an important foundation for realization of teaching objectives. However, since constrained by examination-oriented education for a long term, responsibility of college physical education teachers is in the increasing scarcity, which is not conducive to carry out teaching, and also establishes a negative example for students, for example, students become undisciplined and lazy, highly arbitrary in the classroom, often late and leaving early for no reason and so on. Secondly, lacking quality of teaching is also reflected in lower capacity or the development stagnation of teachers' professional skills. Physical education teachers' professional skills are an indispensable part of overall quality system, and also an important foundation for teachers to show and teach students sports skills. But in fact, most college physical education teachers are too concerned about students' sports skills compliance status, not only unable to conduct teaching design and practice of new sports and activities, and appearing inadequate in the face of the new demands of students, thus not able to achieve the established teaching effect for a long time.

Teachers cannot timely access students' quality of sports and interest, and in teaching design and practice process they seem helpless, because they are unable to really get the support and active participation of students. First, some college physical education teachers lack proper understanding and awareness of the physical education. They think that physical education is only concerned with exercise and assessment of basic activities, and the education level and depth extent are limited. Thus, for a vast majority of students, to participate in sports is only for the sole purpose of passing the compliance test to obtain enough credits. Thus, in college physical education, perfunctory signs are everywhere, and there are many students who just muddle through the course. Secondly, students lack sufficient interest in sports learning. We know that in teaching, only to fully mobilize the interest of students can truly contribute to maximization of the effectiveness of teaching. However, since at present college physical education lacks innovation in terms of mode construction, and therefore cannot meet the needs of students' sports learning, and reduce their interest in sports, so that students, in sports learning, cannot form good autonomy consciousness, and determine the correct orientation of development, resulting in that students become puzzled, confused or depressed in the learning process.

\section{How to Integrate Positive Psychology into College Physical Education}

\section{Strength advantages of physical education in the teaching practice to train cognitive ability of college students.}

Cognitive ability, namely, the ability of human brain to process, store and retrieve information, mainly refers to the ability of people to grasp object constitution, performance and relationship with other things, the driving force of development, direction and basic laws. In general, people can successfully complete activities with mature and stable cognitive ability. Cognitive ability mainly 
includes perception, memory, attention, thinking and imagination and so on. In college physical education, the cognitive ability refers to speed and accuracy of students' reaction to external stimuli, the level of neuromuscular coordination, adaptability degree of body and ideology, thinking ability and judgment ability in collective sports, self-control and emotional development capacity in sports activities, and all of the cognitive positive results can be fully mobilized through sports. However, as the basic property of human, cognitive ability is different due to the particularity of student individuals and teachers only fully investigate and recognize the particularity in teaching activities, and can truly improve the effectiveness of physical education, enable each student to find their own sports, and highlight their cognitive advantages in the sports activity. In the process of guiding students to select sports activity, teachers should be exposed to theoretical and practical knowledge, simple and fine motor movements, individual sports and team sports, body movements and exercise equipment, fitness programs and skills items, strength-based sports and exercise-oriented exercises, etc. First, teachers should let students know relationship between the sports and health, and specific measures may include lectures, videos, discussions, sports and so on, enable students to understand the inherent link from the physiological and psychological mutual effect, and master lifetime sports method from sports knowledge, technology, skills learning, so that students are aware of the importance of sports. Secondly, help students understand their own motor function features, including limb coordination, and movement reactivity, etc. Specific activities may include finger fingering exercises, direction change and circling of arms, pace transformation of legs, the coordination movement exercises of upper and lower limbs, body flipping, jumping, running, etc., so that students understand ties between the nerve dendrites, so as to improve the ability to control self-movement. In addition, teachers can also improve students' ability to comprehend and experience in sports activities through games, contests, appreciation, performances and other ways to make students truly understand and love sports.

\section{Identify group psychological characteristics and orientation, and increase initiative and sense of happiness.}

Positive psychology asks college physical education teachers to pay adequate attention to students' sports psychological state development in the teaching process. They can use mental health assessment tools for students' periodic measurement statistics to determine psychological characteristics and orientation of individuals and group. For example, Symptom Checklist (SCL) compiled by L.R. Derogatis, comprises a total of 90 items, including more extensive psychiatric symptoms, involved with feeling, emotion, thought, consciousness, behavior, habits, relationships, sleeping, eating, etc. Use the checklist to investigate mental health of college students, to get their psychiatric symptoms situation from different angles, and also psychological development variation of students in a particular period. According to related statistics, the test mainly includes somatization, obsessive-compulsive symptoms, interpersonal sensitivity and other factors. Teachers should use group teaching, arrange different sports items, conduct effective integration, and consciously guide students to actively face themselves, understand themselves and transcend themselves. First, somatization factors including cardiovascular, gastrointestinal, respiratory discomfort, headaches, back pain, muscle soreness and anxiety and other symptoms can more fully show students' physical discomfort, and teachers can guide students from breathing, running, health, and daily exercise, etc. Second, symptoms, namely, behaviors and thinking status beyond the control, include, "blank brain", "bad memory", stubborn, excessive pursuit of perfection, hesitant, cautious, etc. Teachers can conduct comprehensive sports activities combining movement and peace to guide students' emotions to make up for their mental deficiencies. In addition, due to mental problems, college students are likely to be sensitive in relationships, for which teachers can take collective activity-based teaching strategies to encourage students to make proper self-expression in group activities and complete the task in self-evaluation and evaluating activities. Specific sports activities include three-person shuttlecock, badminton, mountain climbing, hiking, etc., so that students can enhance self-confidence in sports activities in order to establish a good social interpersonal system. Furthermore, depression and anxiety are common mental contradiction for college students, including a lack of desire for 
activity, unable to rest, nervousness, disappointment, pessimism and irritability and other symptoms, and teachers may use low-intensity but long-time physical education activities to guide students, such as hiking, development training, yoga, qigong and transplant games, to enable students to achieve the effect of endurance exercise, and get enough sense of self-efficacy from successful completion in low-difficulty activities.

\section{Conduct positive learning evaluation.}

In traditional college physical education, teachers tend to focus on results evaluation of students in physical activity, that is, only using the completion performance of movement skills for score evaluation of students, but lacking evaluation on the learning process, and learning attitudes. But positive psychology, as the proactive attitude category, promotes to use positive thoughts to guide and evaluate students, evaluate students' strengths in the results evaluation of sports activities, and pay more attention to the physical and mental state development of students in sports in order to more effectively summarize students' strengths, and actively guide students to right face learning difficulties. Physical education based on positive psychology requires college teachers to take advantage of process evaluation and summative evaluation, relative evaluation and absolute evaluation, qualitative evaluation and quantitative evaluation, and other combined mode, and give full play to advantages of various types of evaluation to improve the evaluation comprehensiveness and objectivity. For example, in the expansive training, create personalized archives for students, and pay attention to students' results and performance in each task, including cooperation degree with others and physical quality, etc.

\section{Conclusions}

In summary, college physical education is faced with reform task at the psychological level, to conform to the times development and general psychological state development of college students. College physical education is given to a new mission, based on improvement of students' physical health condition, also taking up to ease psychological state and eliminating pressure for students. Therefore, college physical education teachers should advance with the times, pay attention to personalized characteristics at the psychological level, redesign and improve the physical education teaching, to achieve the training objective of modern talents.

\section{References}

[1] Kang Jianglong. Reflections on Theoretical Teaching in College Physical Education Courses,Sports Mentorship, 2015 (05): 41-42.

[2] Zhang Yong. On Effect of Dynamic System of Psychology on Psychological Atmosphere in Sports Classroom,Contemporary Physical Science and Technology, 2014 (24): 113-114.

[3] Ma Wenfeng. On Practice of Positive Psychology in College Physical Education Teaching,Intellect, 2014 (30): 101.

[4] Wei Feng. To Improve Mental Function of Physical Education-Positive Psychology,Physical Science and Technology, 2016 (03): 86-87.

[5] Wang Fengqi, Yang Yansheng. Experimental Study on Application of Self-efficacy Sense in College Gymnastics Teaching,Journal of Inner Mongolia University (Natural Science), 2014 (06): 728-737.

[6] Fan Cuihong. Positive Teaching Emotions Characteristics and Regulation of Physical Education Teachers,Combat· Martial Science, 2015 (02): 99-105.

[7] Xia Qingqing. Mental Disorders and Treatment of Physical Education,Stationery and Science \& Technology, 2014 (02): 89-94. 
[8] Yang Ying. On Teaching Concept of Humanistic Psychology in Physical Education,Speed Reading (early), 2014 (05): 67. 